\title{
Level of awareness of dentists about the role of physiotherapy in temporomandibular disorders: a pilot study in Riyadh, Saudi Arabia
}

\author{
Afaf A. M. Shaheen ${ }^{1,2^{*}}$ (D) Hajar Alhajii ${ }^{2}$, Noura Alrajeeb ${ }^{2}$, Ruba Almoammar ${ }^{2}$, Arwa Alyousef ${ }^{2}$,
} Syamala Buragadda ${ }^{2}$ and Ganeswararao Melam²

\begin{abstract}
Background: Physiotherapy (PT) is a distinguished developing profession. It has a crucial role in treating temporomandibular disorders (TMD) as an effective intervention to reduce pain and improve TMD symptoms. Not all dentists are aware of the importance of collaboration with physiotherapists (PTs).Therefore, this study aimed to assess the level of dentists' awareness about the role of PT in treating TMD, identify dentists' willingness to collaborate with PTs, and create collaboration awareness. This cross-sectional study included Saudi dentists in Riyadh. An online survey was used to assess dentist's awareness and their readiness to collaborate with PTs.

Results: Out of 1500 dentists, only 162 participated in the survey. About $46.9 \%$ of dentists were aware that PT can treat TMD. Generally, there was lack of awareness about the benefits of PT among dentists (61.8\%), and 59.9\% was not aware that the evidence suggested that PT can improve TMD symptoms. Only 29\% referred patients to PT. Upon the completion of the survey, almost all dentists (97.5\%) were likely to refer patients with TMD to PT, and $90.7 \%$ of them showed interest to learn more about collaborating with PTs.

Conclusion: The study concluded that, although $46.9 \%$ of the dentists are aware about the role of PT in treating TMD, there is a lack of awareness about the benefits of PT among the majority of them. The study helped to extend the awareness of surveyed dentists about the collaboration and multidisciplinary approach.
\end{abstract}

Keywords: Awareness, Dentists, Temporomandibular joint disorders, Physiotherapy role

\section{Background}

Temporomandibular joint (TMJ) is a synovial joint that helps to move the mandible, and these movements allow opening and closing the mouth. Temporomandibular disorders (TMD) refer to the dysfunction of the TMJ with multifactorial etiologies and pathologies in the orofacial region [1]. TMD are

\footnotetext{
* Correspondence: afaf_pt@yahoo.com

'Department of Basic Sciences, Faculty of Physical Therapy, Cairo University, Cairo, Egypt

${ }^{2}$ Department of Rehabilitation Sciences, College of Applied Medical Sciences, King Saud University, Prince Turki bin Abdulaziz First Road, Riyadh 12371, Saudi Arabia
}

musculoskeletal disorders characterized by tenderness, and clicking within the joint $[2,3]$.

TMJ dysfunction is one of the chronic orofacial pain conditions and is associated with symptoms related to it patient's health related quality of life and $13.3 \%[5,6]$; around $10 \%$ of patients may have severe symptoms $[6,7]$. The prevalence increases with age and is higher in women compared to men $[8,9]$. A recent study done in Saudi Arabia (SA) found that 27.2\% 
of the children and adolescents had at least one TMD diagnosis, and the most common symptom was myofascial pain [10].

The TMD are region-specific heterogeneous groups of common musculoskeletal problems that cause people to seek health care services [11]. Symptoms can be best managed by a multidisciplinary team including PT [3] and is believed that PT plays an important role in the conservative management of TMD [12-14]. Recent evidence [15] showed that PT has a considerable role in treating TMD by increasing jaw opening and mobility and decreasing TMJ pain and inflammation, so it helps to restore function [4].

Physiotherapists (PTs) deal with TMJ as they treat other joints in the body and use different electrotherapy modalities such as electrical stimulation [16-20], ultrasound [21], acupuncture [22-24], and laser therapy [25-27] to reduce pain and inflammation which further promotes healing of tissues. Other conservative approaches including occlusal splint therapy [28], massage [28-30], manual therapy [4,31,32], therapeutic exercises, and home exercises program [33, 34] are used to re-establish the proper flexibility and muscular length/ strength. Therefore, it improves mobility/function.

The awareness about multidisciplinary approach may help health practitioners to refer TMD patients to other specialists for better management of the condition [35], and much better prognosis can be achieved by dentist and PTs collaboration [7]. Up to the authors' knowledge, no studies were done in neither the SA nor other Arab countries to assess the awareness about PT role among dentists. Hence, this study aimed to assess the dentists' awareness level about the role of PT in treating TMD, identify dentists' willingness to collaborate with PTs, and create collaboration awareness.

\section{Methods}

This was a descriptive cross-sectional study involving dentists practicing in both public and private clinics in Riyadh, SA. The study was approved by the College of Medicine Institutional Review Board with reference number (E-19-3768). Dentists who volunteered to participate completed the survey after providing a written informed consent.

\section{Participants}

Information about the licensed dentists working in Riyadh was obtained from the Saudi Commission for Health Specialists (SCFHS) database and Saudi Dental Society (SDS). All 1500 dentists, of both genders with different specialties and years of experience, registered in SCFHS and members of SDS in Riyadh with an active dental license were recruited and invited to participate in the study. Dental students, non-Saudi dentists, and dentists without experience were excluded.

The participants were contacted through e-mails from database of SCFHS and SDS, and the objectives of the study were explained. Meanwhile, an announcement was posted on social media. An online link to the survey was sent via email and social media. The survey was done online through Google Forms between January and April 2019. An educational brochure, with information related to PT's role in treating TMD pain and other symptoms , was available upon the completion of the survey to create/extend the collaboration awareness. Dentists were informed that their participation was voluntary, and any personal information related to their work/job was kept confidential and not published. A reminder email was sent 3 times every 1 week from the initial recruitment email.

\section{Instrumentation}

We adopted the questionnaire from the previous study [7] after taking the permission from the authors. The original survey included 24 questions, with four main domains which include participants' demographics characteristics (seven questions), patients' profile, treatment and referral (12 questions), and questions related to the general knowledge, willingness to collaborate, and interest to learn more about the benefits of the collaborations with PTs (five questions) (Additional file 1). According to the main developer, the survey was revised by dentists, and their feedback was considered during its implantation. Therefore, the survey achieved face and content validity. In the current study, the authors made minor changes in the demographic section where question number six was removed because it is not applicable. The final version of the survey included 23 questions. The time required to complete the survey is 5 to $10 \mathrm{~min}$.

\section{Statistical analysis}

The data was analyzed by using SPSS version 23(IBM SPSS Statistics 23.0). The response to survey questions was analyzed using descriptive statistics. Data were presented as mean \pm standard deviation (SD) for continuous variables and as frequencies, percentages, and ranges for non-continuous variables. Written information provided by the participants was considered and presented. Meanwhile, the general information regarding PT role and referral was calculated and presented.

\section{Results}

Demographic characteristics of the participants

Out of 1500 SCFHS and SDS members, 162 dentists completed the online survey with response rate of $10.8 \%$. The age of the participants ranged from 22 to 60 years 
(mean $33.37 \pm 8.91$ ), and $102(63 \%)$ of the participants were females. Ninety-seven participants $(59.3 \%)$ were with a bachelor's degree, 40 (24.7\%) had a master's degree, and 26 (16\%) had a doctoral degree (PhD). Most of the participants $(69.8 \%)$ were working in governmental hospitals, and $49(30.2 \%)$ were practicing in private clinics \hospitals. The majority (53\%) had $0-5$ years of experience.

Half of the participants were general dentists (51.9\%) followed by other specialties (21.6\%) while only $3.1 \%$ were oral surgeons. About 117 (72.2\%) of the participants had not taken any continuous medical education courses on TMD, and (27\%) of dentists attended workshops related to TMJ, myofascial pain dysfunction syndrome, orofacial pain, basics of TMJ (anatomy, disease, diagnosis, and management), TMD in children and adolescents, occlusion, and minimal invasive management of TMD (Table 1).

\section{TMD patients' information and referral}

About $24.1 \%$ of the dentists reported that 5 to $15 \%$ of their patients had TMJ problems while $4.3 \%$ have not seen TMD patients so far. The most common TMD clinical symptom treated was Bruxism (65\%) followed by jaw muscle tightness. On the other hand, the least common symptoms treated were TMJ degeneration (7\%), and joint hypermobility (6\%). The most common procedures to evaluate TMD were opening and closing the mouth to assess jaw mobility (79.6\%), palpation of TMJ (77.8\%), TMJ sounds (76.5\%), dental occlusion (70.4\%), signs of parafunctional habits (66\%), and palpation of masticatory muscles $(63.6 \%)$. About $75 \%$ of the TMD patients presented a chronic condition during their initial visit.

The most used TMD treatment method was prescribing bite splints/occlusal guards (72.2\%) followed by medication prescription (45.1\%) and occlusion correction/braces (35.8\%). Lastly, $28.4 \%$ of the dentists used other treatment methods including cold/hot packs, massage, range of motion exercises, stretching, advice on stress reduction, psychological support, educating patients about the condition, prosthesis, botox injection, TMJ arthrocentesis, and phototherapy (Fig. 1).

Most dentists often referred TMD patients to oral surgeons $(54.3 \%)$ followed by $41.4 \%$ to other specialists like oral medicine, TMJ, orofacial pain, and less frequently to neurologists, chiropractors, and sleep physicians. Only $29 \%$ of the dentists advised PT referral (Fig. 2). The causes of PT referral were neck postural alteration, e.g., forward head posture (24\%), tenderness of muscles of mastication (23.5\%), neck pain (19.1\%), headache $(17.3 \%)$, and if patient did not get better after dental treatment (17.3\%) (Fig. 3).
Table 1 Demographic characteristic of all participants

\begin{tabular}{|c|c|}
\hline \multicolumn{2}{|l|}{ Variable } \\
\hline Age mean (SD) & $33.37(8.91)$ \\
\hline Gender & $\boldsymbol{N}(\%)$ \\
\hline Female & $102(63)$ \\
\hline Male & $60(37)$ \\
\hline \multicolumn{2}{|l|}{ Education } \\
\hline 2003Bachelor's degree & $97(59.9)$ \\
\hline Master's degree & $40(24.7)$ \\
\hline Doctoral degree & $25(15.4)$ \\
\hline \multicolumn{2}{|c|}{ Years of experience and practicing } \\
\hline $0-5$ & $86(53.1)$ \\
\hline $6-10$ & $30(18.5)$ \\
\hline $11-15$ & $10(6.2)$ \\
\hline $16-20$ & $16(9.9)$ \\
\hline $21-25$ & $10(6.2)$ \\
\hline $26-30$ & $6(3.7)$ \\
\hline $31-35$ & $4(2.5)$ \\
\hline $36-40$ & $0(0)$ \\
\hline $41-45$ & $0(0)$ \\
\hline $46-50$ & $0(0)$ \\
\hline $51-55$ & $0(0)$ \\
\hline More than 55 years & $0(0)$ \\
\hline \multicolumn{2}{|l|}{ Job title } \\
\hline General dentist & $84(51.9)$ \\
\hline Other speciality & $35(21.6)$ \\
\hline Periodontist & $14(8.6)$ \\
\hline Endodontist & $9(5.6)$ \\
\hline Prosthodontist & $8(4.9)$ \\
\hline Orthodontist & $7(4.3)$ \\
\hline Oral surgeon & $5(3.1)$ \\
\hline \multicolumn{2}{|c|}{ Any educational courses on TMD? } \\
\hline No & $117(72.2)$ \\
\hline Yes & $45(27.8)$ \\
\hline
\end{tabular}

$S D$ standard deviation, $N$ number, $\%$ percentage

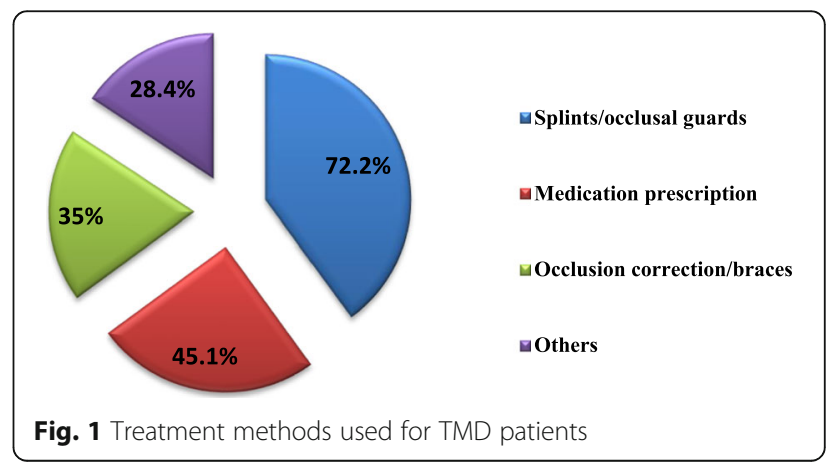




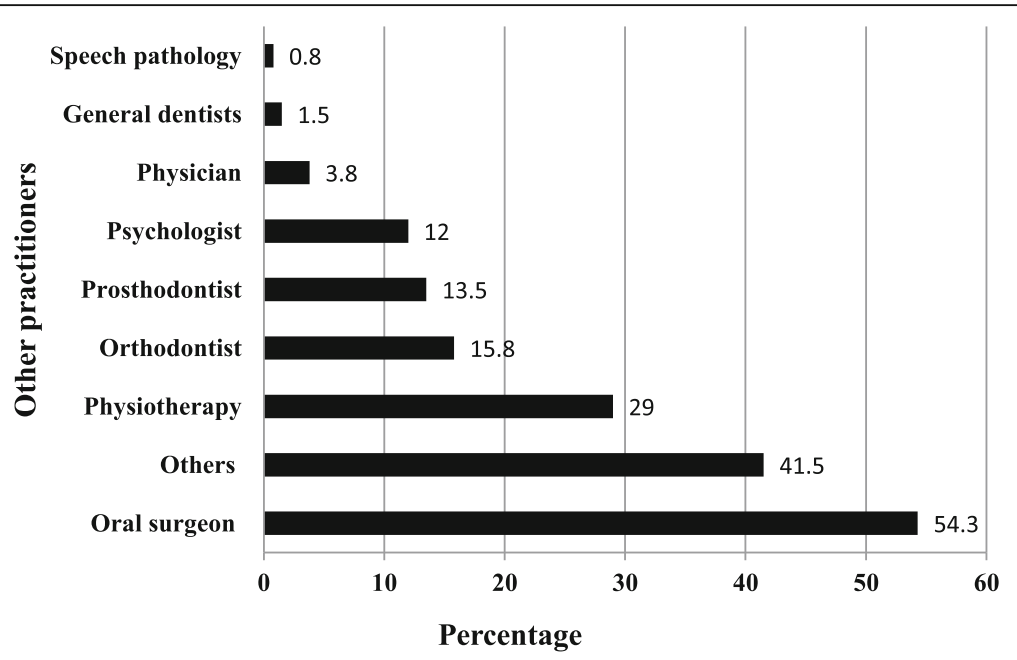

Fig. 2 Dentists' referral to other professionals or practitioners

The main reason of not referring patients to PT was the lack of awareness about the benefits of PT treatment $(61.8 \%)$, while $21.1 \%$ of dentists noted that PT treatment is not essential for the patients and $17.1 \%$ believed that they can manage the patients alone without any referral and unavailability of PT services.

\section{Level of awareness of PT}

Prior to the survey, only 76 dentists (46.9\%) reported that they were aware that PTs can treat patients with TMD while $59.9 \%$ were not aware that the evidence suggests that PT can improve TMD symptoms with oral exercises, manual therapy, and postural reeducation. Furthermore, only $35.8 \%$ of dentists were aware that TMJ pain may also involve cervical spine pain (Fig. 4). On the other hand, after the participation in this study,
97.5\% of dentists was willing to refer patients with TMD to PT when needed. Only one dentist believed that oral maxilofacial specialists can treat the TMD patient instead of PTs. In addition, almost $90.7 \%$ of the participants reported that they were interested to learn about the benefits of collaboration with PTs for treatment of patients with TMD (Table 2).

\section{Discussion}

To the best of authors' knowledge, this is the first study conducted in SA to assess the level of dentists' awareness about the role of PT in treatment of TMD, identify dentists' willingness to collaborate with PTs, and create awareness about the significance of collaboration and the multidisciplinary approach with PTs. The results revealed that about $46.9 \%$ of the dentists are aware about

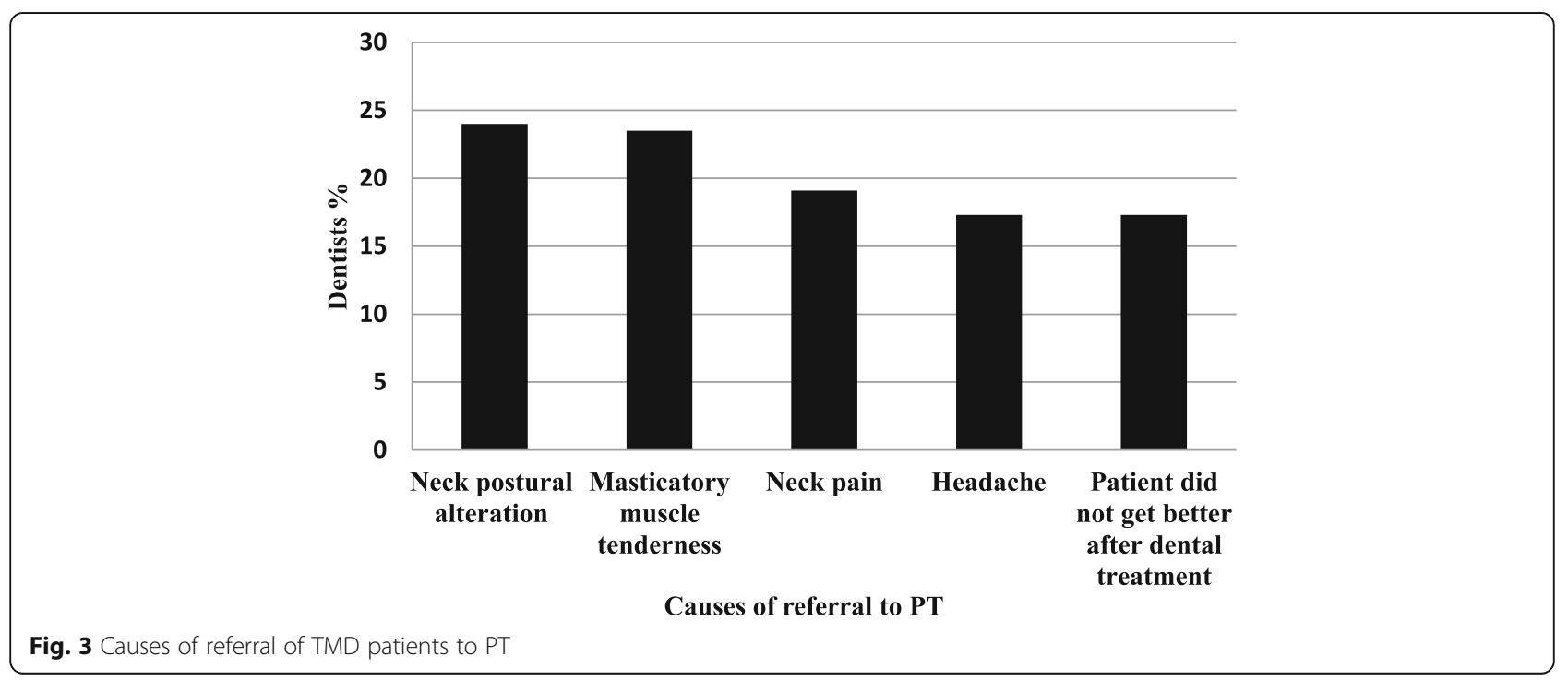




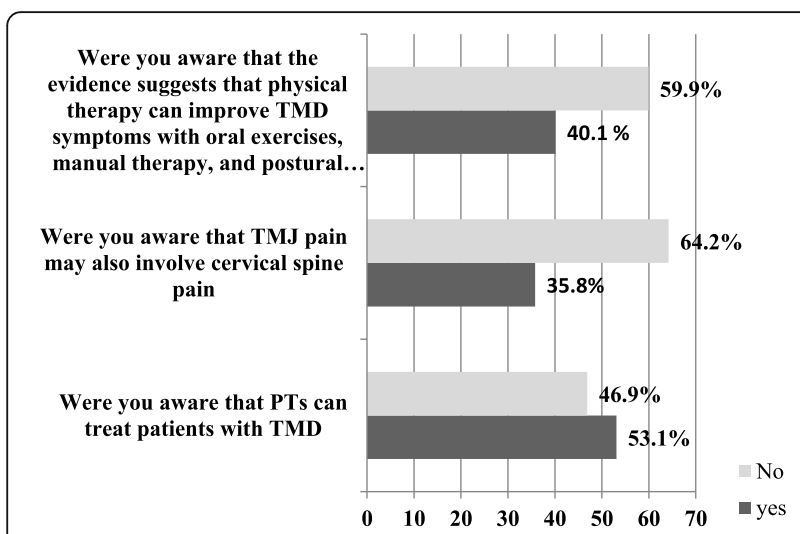

Fig. 4 Dentist's awareness about PT benefits and services prior to the survey

the role of PT in treating TMD; however, there is a general lack of awareness about the benefits of PT among $61.8 \%$, and $59.9 \%$ was not aware that the evidence suggested that PT can improve TMD symptoms. Only 29\% of the dentists advised PT referral. Upon the completion of the survey, almost all dentists (97.5\%) were likely to refer patients with TMD to PT, and $90.7 \%$ of them showed interest to learn more about collaborating with PTs.

The findings of this study must be explained carefully because the response rate was very low (10.8\%), thus the generalizability of the results is doubtful. However, this pilot study explores the current level of awareness among the dentists in Riyadh and may help further increase the level of collaboration between PTs and dentists in the treatment of TMD. The lower response rate reported in this study was very close to Gadotti et al. [7]. The possible causes of that may be lack of time, unwilling to participate in the study, and not all email addresses were updated in the list provided by SCFHS and SDS.

The present study indicates that dentists need to be more educated about PT. The results showed that only 49.9\% had awareness about the role PT in treatment TMD. This proportion was lower than that reported by

Table 2 Dentist's readiness to collaborate with physiotherapist

\begin{tabular}{lll}
\hline Dentists specialty & No $\boldsymbol{N}(\%)$ & Yes $\boldsymbol{N}(\%)$ \\
\hline Endodontist $(N=9)$ & $0(0 \%)$ & $9(100 \%)$ \\
General Dentist $(N=84)$ & $9(11 \%)$ & $75(89 \%)$ \\
Oral Surgeon $(N=5)$ & $2(40 \%)$ & $3(60 \%)$ \\
Orthodontist $(N=7)$ & $0(0 \%)$ & $7(100 \%)$ \\
Periodontist $(N=14)$ & $3(21 \%)$ & $11(79 \%)$ \\
Prosthodontist $(N=8)$ & $0(0 \%)$ & $8(100 \%)$ \\
Other $(N=35)$ & $1(3 \%)$ & $34(97 \%)$ \\
Total $(N=162)$ & $15(9.3 \%)$ & $147(90.7 \%)$ \\
\hline
\end{tabular}

$N$ number, \% percentage a previous study in Florida [7]. The likely reasons of this could be lack of education, knowledge, perception about PT, and lack of experience as the majority of the participants $(59.9 \%$ ) have a bachelor's degree, and $53.1 \%$ is practicing from 5 years or less.

The results showed that the most common TMD symptom was Bruxism followed by jaw muscle tightness, and the least common symptoms were joint hypermobility and TMJ degeneration. In this study, the most common treatment method of TMD was prescribing bite splints or occlusion guards. On the other hand, Wright and North elaborated that patients improved better by bite splints combined with soft tissue massage of masticatory muscles and oral mobilization exercises than bite splints therapy alone [3].

The majority of the participants referred patients with TMD to oral surgeons, and only $29 \%$ of them advised to refer patients to PT. This low rate of referral may be due to (1) the lack of awareness about the benefits of PT among the majority of the participants (61.8\%), (2) $59.9 \%$ were not aware that evidence suggested that PT can improve TMD symptoms with oral exercises, manual therapy, and postural reeducation, (3) $21.1 \%$ of the dentists noted that PT treatment is not essential for the patients, (4) $7.1 \%$ believed that they can manage the patients alone without any referral, and (5) unavailability of PT services.

Further, other possible cause of low referral rate may be the need of accessible PTs with the ability in treating TMD since not all PTs are prepared and confident about giving care to TMD patients. Therefore, the authors actually agree with the suggestion of Gadotti et al. [7] that more education related to TMJ, TMD, and the multidisciplinary approach between dental practitioners and PTs in the management of TMD patients should be also reinforced in all PT programs.

One of the main reasons of PT referral in our study was postural alteration which contradicts with the previous study that considered the cervicogenic headache as an important reason of referral [7]. Kraus reported an association between TMD and head and neck pain [36]. Hence, dentists should be aware of the associative symptoms in TMD patients and refer patients to PT for better outcomes. In fact, awareness about collaboration for better prognosis in TMD patients is not only required for dentists but also needed among PTs. Accordingly, while assessing TMD patients, PTs should be aware of dental problems such as tooth pain and/or dental occlusion problems related to TMJ dysfunction in order to refer the patients to dentists.

Actually, this study helped to increase or extend the level of awareness about the collaboration of dentists with PTs whereby upon the completion of the survey, the participants were asked to read the educational 
brochure containing information related to PT's role in treating TMD. In addition, the responds on the question about the "After participating in this survey, are you more likely to refer a patient with TMD to a physiotherapist when needed?" revealed that about $97.5 \%$ of dentists were willing to refer patients to PT when needed. Besides, almost all the dentists (90\%) were interested to learn more about the benefits of the collaborations with PTs to treat TMD patients.

TMD is a complex condition with many associated symptoms. Hence, multidisciplinary approach is needed for pain management and better prognosis [37]. Therefore, appropriate strategies must be implemented to enhance the knowledge and educate dentists about the various aspects of PT for the improvement of the healthcare system as a whole. This can be achieved by including information about the role of PT in treating TMD in seminars and lectures in the courses of Dentistry Programs to inform them about the importance of interdisciplinary treatment of TMD patients. Besides, relevant authorities and those within the field of PT should be more proactive in raising the awareness and acknowledgment of the developing PT profession in SA for the progression of quality healthcare services in the kingdom.

\section{Study limitations}

Although this is a pilot study done in one province of SA, there are a few limitations. The study included only Saudi dentists in Riyadh. The sample specific nature of the current study may limit generalizability of the findings. Future studies with larger sample (higher response rate) including dentists not only from different regions of SA but also from different ethnicities are needed. In addition, further studies are recommended to evaluate the level of collaborations between PTs and dentists and the treatment benefits achieved by TMD patients from those collaborations.

\section{Conclusion}

This study concludes that even though $46.9 \%$ of the dentists reported that they are aware about the role of PT in treating TMD, there is still a lack of awareness about the benefits of PT among the majority (61.8\%) of dentists in Riyadh. The study made a difference to extend the surveyed dentists awareness about the collaboration and multidisciplinary approach with PT. The collaboration is extremely important for better outcomes, avoiding the misdiagnosing, and lowering the cost.

\section{Supplementary information}

Supplementary information accompanies this paper at https://doi.org/10. 1186/s43161-020-00009-5.

Additional file 1. STROBE_checklist_cross-sectional.

\section{Abbreviations}

PhD: Doctoral degree; PTs: Physiotherapists; PT: Physiotherapy; SA: Saudi Arabia; SCFHS: Saudi Commission for Health Specialists database; SDS: Saudi Dental Society; SPSS: Statistical Package for the Social Sciences;

TMD: Temporomandibular disorders; TMJ: Temporomandibular joint

\section{Acknowledgements}

The authors thank the Research Center of the Female Scientific and Medical Colleges, Deanship of Scientific Research, King Saud University.

\section{Authors contributions}

AS suggested the research idea, helped in data collection, analyzed and interpreted the data regarding the participants' demographics characteristics, patients' profile, treatment and referral, and general knowledge about dentists' awareness and their willingness to collaborate. HA, NA, RA, and AA participated in data collection and analysis and interpretation of data. SB and GM revised the data analysis and they were the major contributors in the writing process. All authors read and approved the final version of the manuscript.

\section{Funding}

The authors confirm that there is no financial support.

\section{Availability of data and materials}

The datasets generated during and/or analyzed during the current study are available from the corresponding author on reasonable request.

\section{Ethics approval and consent to participate}

The study was approved by the College of Medicine Institutional Review Board, King Saud University with reference number (E-19-3768). Dentists who volunteered to participate completed the survey after providing a written informed consent

\section{Consent for publication}

N/A

\section{Competing interests}

The authors declare that they have no competing interests.

Received: 1 May 2020 Accepted: 9 July 2020

Published online: 07 October 2020

\section{References}

1. De Rossi SS, Greenberg MS, Liu F, Steinkeler A. Temporomandibular disorders: evaluation and management. Med Clin. 2014;98(6):1353-84.

2. Wadhwa S, Kapila S. TMJ disorders: future innovations in diagnostics and therapeutics. J Dent Educ. 2008;72(8):930-47.

3. Wright EF, North SL. Management and treatment of temporomandibular disorders: a clinical perspective. J Man Manip Ther. 2009;17(4):247-54.

4. Armijo-Olivo S, Pitance L, Singh V, Neto F, Thie N, Michelotti A. Effectiveness of manual therapy and therapeutic exercise for temporomandibular disorders: systematic review and meta-analysis. Phys Ther. 2016;96(1):9-25.

5. Prodoehl J, Kraus S, Klasser GD, Hall KD. Temporomandibular disorder content in the curricula of physical therapist professional programs in the United States. CRANIO ${ }^{\oplus}$. 2019:1-13.

6. Kraus S, Prodoehl J. Outcomes and patient satisfaction following individualized physical therapy treatment for patients diagnosed with temporomandibular disc displacement without reduction with limited opening: a cross-sectional study. CRANIO ${ }^{\circledR}$. 2019;37(1):20-7.

7. Gadotti IC, Hulse C, Vlassov J, Sanders D, Biasotto-Gonzalez DA. Dentists' awareness of physical therapy in the treatment of temporomandibular disorders: a preliminary study. Pain Res Manag. 2018;2018.

8. Bagis B, Ayaz EA, Turgut S, Durkan R, Özcan M. Gender difference in prevalence of signs and symptoms of temporomandibular joint disorders: a retrospective study on 243 consecutive patients. Int J Med Sci. 2012;9(7): 539-44.

9. Nilsson I-M, List T, Drangsholt M. Prevalence of temporomandibular pain and subsequent dental treatment in Swedish adolescents. J Orofac Pain. 2005;19(2).

10. Al-Khotani A, Naimi-Akbar A, Albadawi E, Ernberg M, Hedenberg-Magnusson B, Christidis N. Prevalence of diagnosed temporomandibular disorders 
among Saudi Arabian children and adolescents. J Headache Pain. 2016; 17(1):41.

11. Shaffer SM, Stuhr SH, Sizer PS, Courtney CA, Brismée J-M. The status of temporomandibular and cervical spine education in post-professional physical therapy training programs recognized by Member Organizations of IFOMPT: an investigation of didactic and clinical education. J Man Manip Ther. 2018;26(2):102-8.

12. Gadotti IC, Lakow A, Cheung J, Tang M. Physical therapists' selfperceived adequacy of entry-level education and their current confidence levels with respect to temporomandibular disorders: a pilot study. CRANIO . 2018:1-8.

13. Melis M. The role of physical therapy for the treatment of temporomandibular disorders. J Orthod Sci. 2013;2(4):113.

14. De Laat A, Stappaerts K, Papy S. Counseling and physical therapy as treatment for myofascial pain of the masticatory system. J Orofac Pain. 2003;17(1)

15. Rashid A, Matthews NS, Cowgill H. Physiotherapy in the management of disorders of the temporomandibular joint-perceived effectiveness and access to services: a national United Kingdom survey. Br J Oral Maxillofac Surg. 2013;51(1):52-7.

16. Núñez SC, Garcez AS, Suzuki SS, Ribeiro MS. Management of mouth opening in patients with temporomandibular disorders through low-level laser therapy and transcutaneous electrical neural stimulation. Photomed Laser Surg. 2006;24(1):45-9.

17. Awan $\mathrm{KH}$, Patil S. The role of transcutaneous electrical nerve stimulation in the management of temporomandibular joint disorder. J Contemp Dent Pract. 2015;16:984-6.

18. de Lima Ferreira AP, da Costa DRA, de Oliveira AIS, Carvalho EAN, Conti PCR Costa YM, et al. Short-term transcutaneous electrical nerve stimulation reduces pain and improves the masticatory muscle activity in temporomandibular disorder patients: a randomized controlled trial. J Appl Oral Sci. 2017:25(2):112-20.

19. Rezazadeh F, Hajian K, Shahidi S, Piroozi S. Comparison of the effects of transcutaneous electrical nerve stimulation and low-level laser therapy on drug-resistant temporomandibular disorders. J Dent. 2017;18(3):187.

20. Monaco A, Cattaneo R, Mesin L, Ortu E, Giannoni M, Pietropaoli D. Dysregulation of the descending pain system in temporomandibular disorders revealed by low-frequency sensory transcutaneous electrical nerve stimulation: a pupillometric study. PLoS One. 2015;10(4):e0122826.

21. Ucar M, Sarp Ü, Koca I, Eroğlu S, Yetisgin A, Tutoglu A, et al. Effectiveness of a home exercise program in combination with ultrasound therapy for temporomandibular joint disorders. J Phys Ther Sci. 2014;26(12):1847-9.

22. Noiman M, Garty A, Maimon Y, Miller U, Lev-Ari S. Acupuncture for treating temporomandibular disorder: retrospective study on safety and efficacy. J Acupunct Meridian Stud. 2010;3(4):260-6.

23. Hunter EK. Integration of rehabilitation and acupuncture in the treatment of a professional musician with temporomandibular joint dysfunction. Acupunct Med. 2011;29(4):298-301.

24. Itoh K, Asai S, Ohyabu H, Imai K, Kitakoji H. Effects of trigger point acupuncture treatment on temporomandibular disorders: a preliminary randomized clinical trial. J Acupunct Meridian Stud. 2012:5(2):57-62.

25. Kato MT, Kogawa EM, Santos CN, Conti PCR. TENS and low-level laser therapy in the management of temporomandibular disorders. J Appl Oral Sci. 2006;14:130-5.

26. Seifi M, Ebadifar A, Kabiri S, Badiee MR, Abdolazimi Z, Amdjadi P. Comparative effectiveness of low level laser therapy and transcutaneous electric nerve stimulation on temporomandibular joint disorders. J Lasers Med Sci. 2017:8(Suppl 1):S27.

27. Xu G-Z, Jia J, Jin L, Li J-H, Wang Z-Y, Cao D-Y. Low-level laser therapy for temporomandibular disorders: a systematic review with meta-analysis. Pain Res Manag. 2018;2018.

28. de Paula Gomes CAF, Politti F, Andrade DV, de Sousa DFM, Herpich CM, Dibai-Filho AV, et al. Effects of massage therapy and occlusal splint therapy on mandibular range of motion in individuals with temporomandibular disorder: a randomized clinical trial. J Manip Physiol Ther. 2014;37(3):164-9.

29. Capellini VK, de Souza GS, de Faria CRS. Massage therapy in the management of myogenic TMD: a pilot study. J Appl Oral Sci. 2006;14(1): 21-6.

30. Biasotto-Gonzalez DA, Bérzin F. Electromyographic study of patients with masticatory muscles disorders, physiotherapeutic treatment (massage). Br J Oral Sci. 2004;3(10):516-21.
31. Kalamir A, Pollard H, Vitiello AL, Bonello R. Manual therapy for temporomandibular disorders: a review of the literature. J Bodyw Mov Ther 2007;11(1):84-90.

32. Cuccia A, Caradonna C, Annunziata V, Caradonna D. Osteopathic manual therapy versus conventional conservative therapy in the treatment of temporomandibular disorders: a randomized controlled trial. J Bodyw Mov Ther. 2010;14(2):179-84.

33. Machado BCZ, Mazzetto MO, Da Silva MAMR, de Felício CM. Effects of oral motor exercises and laser therapy on chronic temporomandibular disorders: a randomized study with follow-up. Lasers Med Sci. 2016;31(5):945-54.

34. Michelotti A, de Wijer A, Steenks M, Farella M. Home-exercise regimes for the management of non-specific temporomandibular disorders. J Oral Rehabil. 2005:32(11):779-85.

35. Kiran MS, Joy ET, Vidya S, Krishna S, Hegde S. Attitude and awareness of temporomandibular joint disorders among general dental practitioners in Southern India. J Adv Clin Res Insights. 2016;3(3):72-5.

36. Kraus S. Temporomandibular disorders, head and orofacial pain: cervical spine considerations. Dent Clin. 2007;51(1):161-93.

37. Schiffman E, Ohrbach R, Truelove E, Look J, Anderson G, Goulet J, et al. International RDC/TMD Consortium Network, International association for Dental Research; Orofacial Pain Special Interest Group, International Association for the Study of Pain. J Oral Facial Pain Headache. 2014;28(1):627.

\section{Publisher's Note}

Springer Nature remains neutral with regard to jurisdictional claims in published maps and institutional affiliations.

\section{Submit your manuscript to a SpringerOpen ${ }^{\circ}$ journal and benefit from:}

- Convenient online submission

- Rigorous peer review

- Open access: articles freely available online

- High visibility within the field

- Retaining the copyright to your article

Submit your next manuscript at $\boldsymbol{\nabla}$ springeropen.com 